\title{
Calidad de los bosques ribereños y perspectivas de restauración en un río de la Patagonia Andina, Argentina
}

\author{
Adriana M. Kutschker ${ }^{\varpi}$; Gabriela Papazian; Oscar A. Martínez \& Nancy Ibañez \\ Facultad de Ciencias Naturales y Ciencias de la Salud, Universidad Nacional de la Patagonia San Juan Bosco, Sede Esquel. \\ Chubut, Argentina.
}

\begin{abstract}
RESUMEN. Las riberas son uno de los aspectos más importantes a considerar para cuantificar y calificar el estado ecológico de los ecosistemas acuáticos. El objetivo de este trabajo fue evaluar la calidad de los bosques de ribera y las perspectivas de restauración del río Carrileufu, desde sus nacientes en lago Cholila hasta su desembocadura en lago Rivadavia, NO de Chubut. Se incluyeron diez tramos sobre el curso principal y dos ubicados en uno de sus afluentes, el río Pedregoso. Se realizó una caracterización física y ecológica a nivel ecosistémico y a escala de tramo, y se registraron variables ambientales, atributos de la comunidad vegetal y procesos geomorfológicos activos e inactivos en el entorno fluvial. Se aplicó el índice QBRp en doce tramos de $100 \mathrm{~m}$ de longitud, sometidos a uno o más usos de la tierra (i.e., plantaciones de exóticas, urbanización, actividad agropastoril, recreativo-turística). Con la información relevada como insumo, se aplicó un análisis multicriterio que permitió definir la factibilidad de la restauración y el manejo más adecuado para cada tramo del río. Los valores del índice QBRp variaron entre calidad de ribera intermedia a muy buena, sin tramos con calidad mala o pésima. Se observó una relación inversa entre la calidad de los bosques de ribera y la superposición e intensidad de usos. En cuanto a las perspectivas de restauración, tres tramos del río presentaron una factibilidad 'sencilla', cuatro de ellos 'moderada' y cinco 'difícil', con distintos tipos de manejo indicados para su recuperación, que van desde la conservación/regeneración, restauración, restauración compleja/revegetación, hasta rehabilitación de los tramos más degradados.
\end{abstract}

[Palabras clave: ambiente acuático, Carrileufu, estado ecológico, manejo, QBRp]

\begin{abstract}
Aвstract. Quality assessment and restoration perspectives of riparian forest in an Andean river from Patagonia, Argentina. The river banks are one of the most important aspects to quantifying and qualifying the ecological status of aquatic ecosystems. An evaluation of the quality of the riparian forests and the restoration perspectives of the Carrileufu river, from its sources in Cholila lake to its mouth in Rivadavia lake, NW of Chubut was raised. Two stretches of the tributary Pedregoso river were also included. A physical and ecological characterization was carried out at the ecosystem level and at the stretch level, registering environmental variables, plant community attributes and the active and inactive geomorphological processes in the river environment. The QBRp index was applied in twelve stretches of $100 \mathrm{~m}$ in length, subject to one or more land uses (i.e., exotic plantations, urbanization, agriculture, livestock, recreational-tourist activity). A multicriteria analysis was applied, with the information relieved as input, which allowed defining the feasibility of restoration and the most appropriate management for each stretch of the river. The values of the QBRp index varied between intermediate to very good river bank quality, without bad or bad quality; observing an inverse relationship between the riparian forest quality and the overlap and intensity of land uses. Regarding to restoration prospects, three stretches presented a feasibility defined as 'simple', four of them 'moderate and five showed a 'difficult' one. Different management ways for their recovery were established, ranging from conservation/regeneration, restoration, complex restoration/revegetation, and even rehabilitation of the more degraded stretches.
\end{abstract}

[Keywords: aquatic environment, Carrileufu, ecological status, management, QBRp]

\section{INTRODUCCIÓN}

Los ambientes ribereños son una parte esencial de los ecosistemas fluviales. Presentan una estructura compleja y heterogénea y actúan como cinturón de protección para el río, atenuando los potenciales efectos negativos derivados de las presiones y amenazas del entorno (Allan 2004). En esta interfaz ocurren interacciones físicas, químicas y biológicas que le confieren propiedades particulares al suelo y a la vegetación (Gregory et al. 1991). La vegetación de ribera constituye, por lo tanto, uno de los elementos fundamentales en la regulación de los procesos fluviales.

Los bosques de ribera crecen en las márgenes de los ríos o sus llanuras de inundación y presentan una extensión espacial que varía longitudinal y lateralmente a lo largo de la red dedrenaje(Naimanetal.1998), redistribuyendo materiales a través del paisaje y brindando funciones hidrológicas y ecosistémicas sustanciales (Mander et al. 2005). La cobertura 
vegetal regula la fuerza de las crecidas, con lo cual estabiliza las márgenes del río y reduce su erosión. En sitios con suelos permeables y bajo coeficiente de escorrentía aumenta la capacidad de infiltración y retención de agua, lo que permite la recarga de los acuíferos (Elosegi and Díez 2009). El bosque amortigua el ingreso de sedimentos y contaminantes a los cursos de agua y disminuye la temperatura del agua por sombreo, lo que repercute en la estructura y la dinámica de los diferentes niveles tróficos (Sabater et al. 2009). Asimismo, la caída de troncos aumenta la complejidad estructural del cauce y favorece tanto la retención de partículas como la creación de nuevos hábitats (Swanson et al. 2017). Ofrece también ambientes propicios para actividades recreativas y otros usos de la tierra (DeFries et al. 2004; MacFarlane et al. 2018). En este contexto, mantener la integridad de los bosques ribereños contribuye a conservar la calidad y biodiversidad de los ecosistemas acuáticos y su entorno.

A pesar de la importancia de los ecosistemas ribereños y de los cursos de agua que le dan origen, estos ambientes están entre los paisajes más degradados por la acción del ser humano (Dudgeon et al. 2006; Scott et al. 2018). Además de la expansión urbana, en la Patagonia se desarrollan distintas actividades (e.g., extracción forestal, agricultura, ganadería extensiva e intensiva, plantaciones con coníferas exóticas) que de manera directa o indirecta afectan la integridad ecológica de los sistemas acuáticos (Miserendino et al. 2008, 2011; Kutschker et al. 2009). A lo ya descripto se suma la dificultad que existe en la Argentina respecto a la interpretación y demarcación en terreno de la línea de ribera (Maurín 2014), con las consecuentes implicancias que esto tiene en el uso y la conservación de los ambientes ribereños. En la actualidad, en varios países se llevan adelante estudios orientados a restaurar aquellos corredores ribereños afectados por los diversos usos de la tierra (Meli 2013; Boudell et al. 2015; Scott et al. 2018). Existen diferentes formas de manejo en estos ecosistemas (e.g., rehabilitación, revegetación, regeneración, restauración), tendientes a recuperar los procesos fluviales, incrementar la resiliencia del ecosistema acuático, establecer un sistema compatible con el uso de la tierra o recuperar el paisaje natural (Valero et al. 2014). La elección de la intervención más adecuada va a depender de las condiciones del entorno inmediato al río (dimensión transversal) y del estado de los tramos situados aguas arriba y abajo del tramo a tratar (dimensión longitudinal).

Es claro que los valores y las funciones que presentan los bosques de ribera los hacen excelentes indicadores de la gestión del territorio y son útiles como elemento clave para la calificación del estado ecológico de los ríos. En la actualidad existen numerosos métodos para valorar de forma rápida y sencilla las riberas; el índice de Calidad del Bosque de Ribera (QBR), propuesto por Munné et al. (1998, 2003), es uno de los más usados y adaptados en diferentes regiones de América (Fernández et al. 2016). En particular, el índice QBRp es una adaptación propuesta por Kutschker et al. (2009) para ríos y arroyos de la cordillera patagónica. Si bien la aplicación de este tipo de índices se ha extendido a nivel mundial y se han conectado los resultados de las evaluaciones de la calidad de los bosques de ribera con la generación de pautas de manejo en función del estado ecológico de las mismas (CHS 2008; Valero et al. 2014; MacFarlane et al.2018), esta tarea es incipiente en la Argentina.

El Carrileufu es un río andino-patagónico que a lo largo de todo su recorrido se encuentra sometido a múltiples usos de la tierra y que, además de su belleza paisajística, constituye la vía de ingreso al Parque Nacional Los Alerces, declarado Sitio de Patrimonio Mundial de la Humanidad por la UNESCO en 2017. En virtud de su ubicación estratégica desde el punto de vista de la conservación, en este trabajo se plantearon los siguientes objetivos: 1) caracterizar los factores y procesos ecosistémicos asociados a este sistema fluvial, integrando las dimensiones biofísicas y de uso del territorio, 2) evaluar cómo afectan los diferentes usos de la tierra y su sinergia en la calidad de los bosques de ribera y 3) aplicar un análisis multicriterio para evaluar las perspectivas de restauración de las riberas en cada tramo del río y, en función de ello, proponer el manejo más adecuado. Además de constituir una línea de base para el monitoreo a mediano y largo plazo del estado ecológico de los ambientes ribereños, del impacto de disturbios naturales o de intervenciones antrópicas, este estudio aporta un abordaje que podrá ser aplicado a otros sistemas fluviales similares para su adecuada gestión y manejo. 


\section{MATERIALes y MÉTOdOS}

\section{Área de estudio}

El estudio se realizó en las riberas del río Carrileufu, ubicado en el NO de la provincia de Chubut, Argentina, entre los $42^{\circ} 27^{\prime}-42^{\circ} 33^{\prime} \mathrm{S}$ y los $71^{\circ} 31^{\prime}-71^{\circ} 38^{\prime} \mathrm{O}$, a una altitud promedio de 538 m s. n. m. La subcuenca del río Carrileufu se ubica en el sector septentrional de la cuenca Futaleufu-Yelcho, la cual abarca una superficie aproximada de $7630 \mathrm{~km}^{2}$ (SSRH 2002) y desagua en el Océano Pacífico. Su cabecera es alimentada por los arroyos Tigre, Blanco y Villegas, que aportan sus aguas al lago Cholila, de cuya margen este nace el río Carrileufu. A pocos metros de su naciente se le une su principal emisario, el río Pedregoso; luego de recorrer varios kilómetros, desemboca en el sector nororiental del lago Rivadavia. A $2.5 \mathrm{~km}$ de dicho punto se ingresa, por el límite Norte, al Parque Nacional Los Alerces (Figura 1). El Carrileufu es un río de $28 \mathrm{~km}$ de extensión, de aguas profundas y claras, con un caudal medio anual de $48.68 \mathrm{~m}^{3} / \mathrm{s}$, un caudal medio máximo en diciembre de $65.16 \mathrm{~m}^{3} / \mathrm{s}$ y de estiaje de 28.17 $\mathrm{m}^{3}$ /s (Calderón 2007).

El valle por donde discurre el río Carrileufu está compuesto por dos segmentos principales: uno ubicado al norte, conectado con el lago Cholila, que tiene orientación NO-SE y otro, con orientación NE-SO, que culmina en el lago Rivadavia. El fondo de este valle suele superar los $2 \mathrm{~km}$ de ancho y su topografía incluye sectores planos, en los que dominan los depósitos fluviales y las lomadas bajas de rocas volcánicas y graníticas suavizadas por la erosión glacial. Este amplio fondo de valle está limitado por laderas ocasionalmente muy inclinadas sobre las que se desarrollaron abanicos aluviales amplios. Por toda el área se distribuye una cubierta de depósitos de tefra (ceniza volcánica que ha contribuido al desarrollo del suelo) y de sedimentos glaciales de distinta granulometría. Los valles de los ríos Pedregoso y Carrileufu, antes de desembocar en los lagos Cholila y Rivadavia, respectivamente, constituyen auténticos humedales de alto valor productivo (Martínez 2007).

El clima de la zona es templado-frío, con precipitaciones pluviales y nivales concentradas en la estación invernal; así, se establece un régimen hídrico de descarga concentrado en dos picos máximos al año: el primero, coincidente con las fuertes precipitaciones invernales, y el segundo, en primavera, a causa del derretimiento de hielo y nieve en las montañas (Coronato and del Valle 1988). La vegetación característica del área es el bosque de transición, con predominio de ñire (Nothofagus antarctica), coihue (N. dombeyi), ciprés de la cordillera (Austrocedrus chilensis), maitén (Maytenus boaria) y un sotobosque arbustivo y herbáceo rico en especies nativas (Diostea juncea, Discaria chacaye, Schinus patagonicus, Lomatia hirsuta, Berberis spp., Baccharis spp.) (Ezcurra and Brion 2005).

En general, los principales usos de la tierra están relacionados con la cría de bovinos en forma extensiva, con excepción de estancia Los Murmullos, donde tiene lugar una actividad agropecuaria importante, con ganadería intensiva y pasturas en gran parte de su superficie. Otros usos comunes en la zona están relacionados con la actividad forestal (aserraderos), extracción de leña y áridos y actividades turístico-recreativas. A lo largo de todo el recorrido del río Carrileufu, aunque de manera dispersa, hay asentamientos de pobladores, campings, lodges de pesca y el barrio cerrado La Frontera, desarrollo inmobiliario privado de $\sim 125$ ha. A pocos kilómetros del acceso Norte del PN Los Alerces se emplaza la Villa Lago Rivadavia, población rural ubicada en la margen izquierda del río (Figura 1).

\section{Diseño de muestreo}

El muestreo se realizó en época de estiaje entre los años 2014 y 2015. Se seleccionaron 12 tramos de ribera de $100 \mathrm{~m}$ de longitud y un ancho promedio de $45 \mathrm{~m}$. Diez tramos se ubicaron sobre el río Carrileufu desde sus nacientes en el lago Cholila hasta su desembocadura en el lago Rivadavia, y se incluyeron, además, dos tramos del río Pedregoso para evaluar el posible efecto diferido de actividades realizadas en uno de los afluentes más importantes del Carrileufu. Se eligieron al menos dos tramos con el mismo uso de la tierra (i.e., forestal, agrícola, ganadero, turístico-recreativo, urbanización), algunos de los cuales presentaron superposición de usos.

En cada sitio se midieron las variables físicas y ecológicas ancho del lecho mojado (equivalente a lecho fluvial) y lecho seco (equivalente a la planicie de inundación activa anual), tipo de sustrato de la ribera (teniendo en cuenta el tamaño del material inorgánico que lo compone [i.e., bloque, guijón, guijarro, gravas, arenas, limo y arcilla]), velocidad 


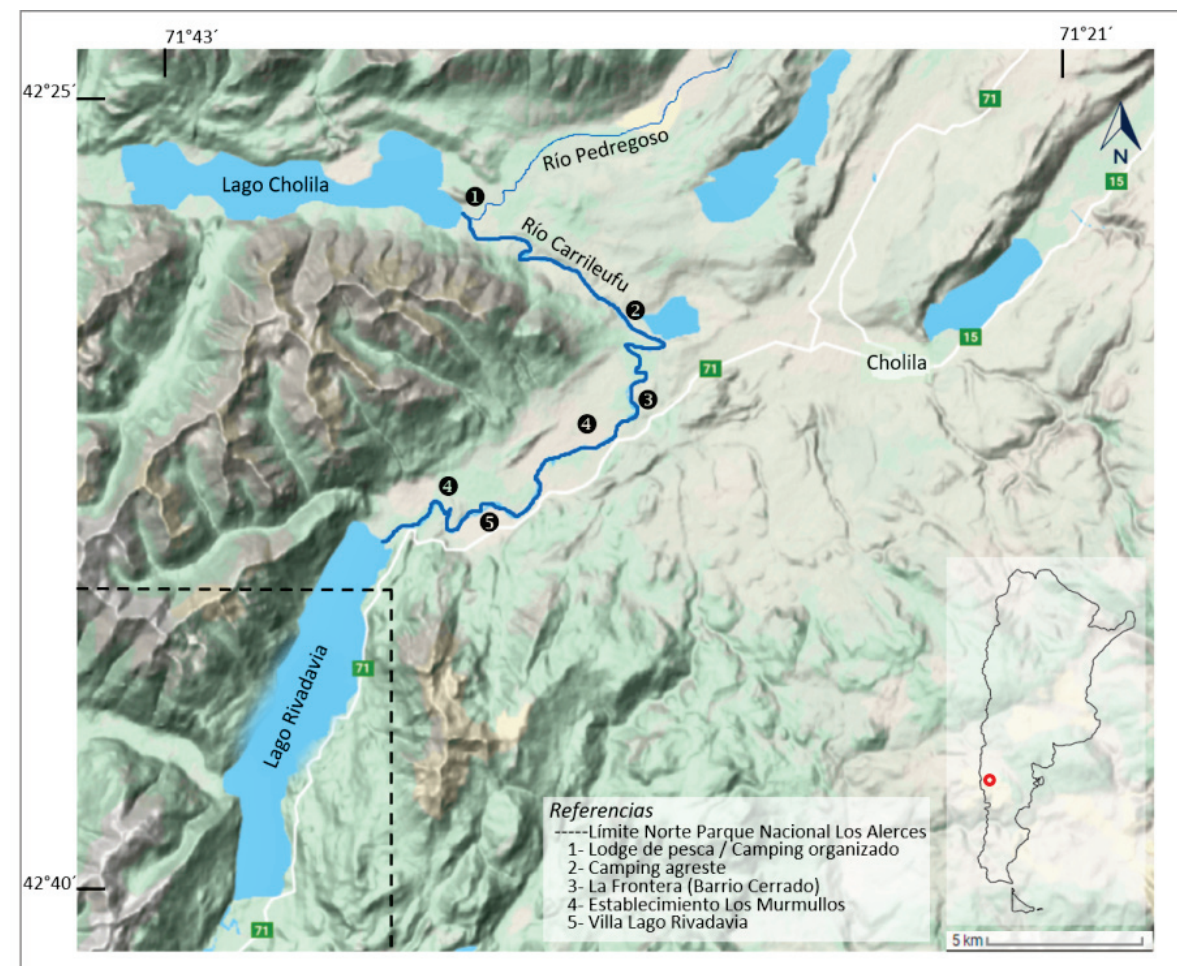

Figura 1. Ubicación geográfica del río Carrileufu y su tributario río Pedregoso en el Departamento Cushamen, al NO de la provincia de Chubut. Se indica el límite NE del PNLA y algunos lugares visitados durante el estudio.

Figure 1. Geographic location of Carrileufu and Pedregoso rivers in Cushamen Department, Nortwestern Chubut. The NE limits of the PNLA and some places visited during the study are indicated.

superficial del flujo de agua $(\mathrm{m} / \mathrm{s})$ (Gordon et al. 2004), pendiente de la ribera (expresada en porcentaje), agentes y procesos geomórficos activos, usos de la tierra asociados a las riberas y en los terrenos adyacentes a las mismas. En gabinete se estimó la tipología del canal fluvial, para lo cual se calculó el grado de sinuosidad (SI) utilizando el método de Brice (1964). El mismo se determinó sobre una imagen satelital de Google Earth Pro versión 7.3.2 y se clasificó según una de las tres categorías propuestas por el Protocolo HIDRI (2006).

Para caracterizar la vegetación ribereña se registró en cada tramo del río la composición y la riqueza de especies. Para la identificación de las plantas se utilizó la colección de Flora Patagónica (Correa 1978-2009) y el catálogo de Plantas Vasculares del Cono sur (Zuloaga et al. 2008). Se estimó in situ la cobertura total y por especie y el porcentaje de sustrato desprovisto de vegetación (SD), analizando cada ribera por separado; esta determinación se realizó de manera subjetiva y considerando que la cobertura podía superar el $100 \%$. Con los datos de riqueza y abundancia relativa se calculó para cada sitio el índice de diversidad de Shannon-Wiener $\left(\mathrm{H}^{\prime}\right)$ y la equitatividad (H'/H'máx.).

Se evaluó la calidad de los bosques de ribera del río Carrileufu y su afluente río Pedregoso mediante el índice QBRp (Kutschker et al. 2009), modificado de Munné et al. (1998). Este índice se centra en aspectos fundamentales de la vegetación ribereña, que se agrupan en cuatro apartados que analizan el grado de cobertura vegetal del corredor ribereño, la estructura de la vegetación, la naturalidad y complejidad de la cubierta vegetal y el grado de alteración del canal fluvial. Cada uno de estos apartados descriptos se evalúa con una puntuación que oscila entre 0 y 25 . La suma de los mismos corresponde al valor del índice QBRp, que varía entre 0 (calidad pésima) y 100 (calidad muy buena). Los valores obtenidos para cada apartado del índice en los distintos tramos del río se registraron en una planilla de campo.

\section{Análisis de los datos}

Se estimó el coeficiente de correlación de Pearson para cada par de variables 
ambientales y ecológicas consideradas en el estudio (i.e., altitud, ancho de lecho mojado y seco, velocidad del agua, pendiente de ambas riberas, cobertura vegetal por ribera y total, suelo desnudo, QBR total y por apartado y cantidad de usos de la tierra), a los efectos de obtener una medida de la magnitud de la asociación lineal entre ellas. Se aplicó una técnica de ordenación, para lo cual se descartaron las variables ancho lecho seco, cobertura por ribera y los apartados de QBRp: calidad, cubierta y naturalidad, ya que mostraron una fuerte correlación entre sí ( $r>0.80)$. Con las variables seleccionadas se efectuó un Análisis de Hill-Smith (AHS) (Hill and Smith 1976) utilizando el software $\mathrm{R}$ versión 3.2.3.

\section{Factibilidad de restauración}

Para evaluar las acciones de manejo a implementar en los distintos tramos del río en función del estado ecológico de sus riberas se aplicó un análisis multicriterio (modificado de CHS 2008). Para ello se definieron categorías de estado a partir de la consideración conjunta de la calidad del bosque de ribera según el Índice QBRp y el tipo de uso al que está sometido cada tramo del río. Se calificaron de forma positiva, para cada tramo, los siguientes aspectos considerados como valores para su conservación o potencialidades de restauración.

Calidad de las riberas. Se asignó una puntuación del 1 al 5 en función de los valores de QBRp obtenidos en cada tramo: (1) <25; (2) >25-50; (3) >50-70; (4) >70-90; (5) $>90$, entendiendo que los valores mayores puntúan mejor los sectores de mayor calidad o integridad ecológica.

Proximidad a tramos con riberas bien conservadas. Se suma un punto a aquellos tramos que incluyan fragmentos de vegetación de ribera en buen estado o que linden con otras riberas en estado bueno o muy bueno.

Hábitats de interés comunitario. Se suma un punto a los tramos donde estén presentes tres o más hábitats ribereños de interés comunitario, definidos en este trabajo como aquellos sitios que brindan bienes y servicios ecosistémicos directos y accesibles para la población en general.

Asimismo, se valoraron de manera negativa las presiones a las que estaban sometidos los distintos tramos del río, y son las que limitan las posibilidades de restauración de sus riberas. Por cada presión negativa de los seis tipos considerados se restó un punto: regulación de caudales, extracciones (áridos, agua, leña, madera), alteraciones hidromorfológicas, contaminación difusa, contaminación puntual y otras alteraciones como la presencia de especies exóticas invasoras.

Una vezevaluados los aspectos anteriormente descriptos se establecieron tres categorías de estado: Bueno, Intermedio y Deficiente. A cada categoría se le asignó una puntuación y su correspondiente factibilidad de restauración y recomendación de manejo (CHS 2008), según se detalla en la Tabla 1.

Las formas de manejo consideradas se definieron según SER (2004) y CHS (2008): a) conservación [protección de las áreas evitando cualquier daño o deterioro futuro en el ecosistema acuático], b) regeneración [intervención de pequeña envergadura sobre el medio para que el ecosistema sea capaz de mejorar su calidad actual], c) rehabilitación [intervención del área más o menos intensa en función de las causas de la degradación, que busca reparar los procesos, la productividad y los servicios de un ecosistema], d)

Tabla 1. Categorías de estado, factibilidad de restauración y recomendaciones de manejo de ambientes ribereños (CHS 2008).

Table 1. Status categories, restoration feasibility and management recommendations for riparian environments (CHS 2008).

\begin{tabular}{cccc}
\hline Categoría de Estado & Puntuación & Factibilidad de Restauración & Manejo Recomendado \\
\hline Estado bueno & $7-6$ & Muy fácil & Conservación \\
Estado intermedio & 5 & Fácil & Conservación/Regeneración \\
Estado deficiente & $1-3$ & Moderada & Restauración \\
& 2 & Difícil & Restauración compleja/Revegetación \\
& $-1--4$ & Difícil & Rehabilitación \\
\hline
\end{tabular}


restauración [similar a la rehabilitación, pero incluye además el restablecimiento de la comunidad biótica preexistente en términos de composición de especies y estructura de la comunidad, retornando a un ecosistema lo más natural posible o equivalente], e) revegetación [acondicionamiento del medio en áreas muy degradadas, a partir del establecimiento de una o unas pocas especies] y f) remediación [intervención en áreas con niveles de degradación severa].

\section{Resultados}

Las variables físicas con mayores diferencias entre sitios correspondieron al ancho del lecho seco; los tramos del río Pedregoso (P1 y P2) fueron los que presentaron la mayor relación lecho seco/lecho mojado. Esa relación fue inferior en todos los tramos del Carrileufu. La pendiente también fue variando a lo largo del río, con riberas más suaves hacia su desembocadura, en los sitios C7 y C10; los restantes tramos se caracterizaron por desniveles en las márgenes, con pendientes entre $\operatorname{los} 17^{\circ}$ y $37^{\circ}$. La morfología del cauce fluvial resultó sinuosa en la mayoría de los tramos, en tanto sólo tres de ellos resultaron meandriformes (Tabla 2).
Entre las geoformas registradas se pueden mencionar las extensas planicies de inundación y bancos de sedimentos en los tramos ubicados en el arroyo Pedregoso; terrazas fluviales extendidas a lo largo del Carrileufu, abanicos aluviales, meandros abandonados y un delta incipiente en la desembocadura del Carrileufu en el lago Rivadavia (Figura 3). El material constitutivo del sustrato de sistema fluvial presentó granulometrías variadas, en distintas proporciones. Se observaron escasos bloques y guijones y en general, los tamaños de clastos más abundantes correspondieron al rango guijarro y limo, con arena y limo entre los dominantes.

Se registraron un total de 45 especies de plantas vasculares, con el ñire nativo (Nothofagus antarctica) y el sauce exótico (Salix spp.) presentes en todos los tramos. Otras especies muy frecuentes fueron rosa mosqueta (Rosa rubiginosa), lupino (Lupinus sp.), calafate (Berberis microphylla) y gramíneas (Poaceae) (Anexo 1). El 57.7\% de las plantas relevadas resultaron nativas y de ellas, el 20\% correspondió a especies arbóreas, el 50\% a arbustivas y el 30\% a herbáceas. Del total de especies exóticas, $84 \%$ estuvo representado por herbáceas. En relación al hábito de la vegetación ribereña, resultó variable la

Tabla 2. Ubicación geográfica y datos de las variables físicas de los distintos tramos analizados en ríos Pedregoso y

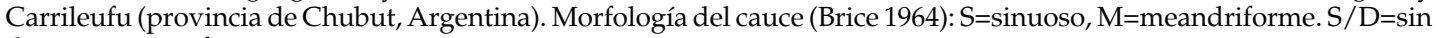
determinar por fuertes vientos.

Table 2. Location and physical variable values of the different stretches from Pedregoso and Carrileufu rivers (province of Chubut, Argentina). River channel morphology (Brice 1964): $S=$ sinuous, $M=$ meandering, $S / D=$ not determined due to strong winds.

\begin{tabular}{|c|c|c|c|c|c|c|c|c|c|c|}
\hline \multirow{2}{*}{$\begin{array}{c}\text { Sitios } \\
\text { Pedregoso }\end{array}$} & \multirow{2}{*}{$\begin{array}{c}\text { Código } \\
\text { P2 }\end{array}$} & \multicolumn{2}{|c|}{ Coordenadas geográficas } & \multirow[t]{2}{*}{ 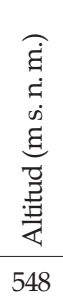 } & \multirow[t]{2}{*}{ 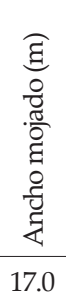 } & \multirow[t]{2}{*}{ 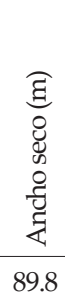 } & \multirow[t]{2}{*}{ 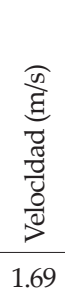 } & $\begin{array}{l}\text { Pen } \\
\text { ribe }\end{array}$ & $\begin{array}{l}\text { ente } \\
s\left({ }^{\circ}\right)\end{array}$ & \multirow[t]{2}{*}{ 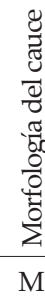 } \\
\hline & & $42^{\circ} 27^{\prime} 53.4^{\prime \prime} \mathrm{S}$ & $71^{\circ} 35^{\prime} 26.2^{\prime \prime} \mathrm{O}$ & & & & & 20 & 26 & \\
\hline Pasarela Pedregoso & P1 & $42^{\circ} 28^{\prime} 05.0^{\prime \prime} \mathrm{S}$ & $71^{\circ} 35^{\prime} 54.0^{\prime \prime} \mathrm{O}$ & 544 & 14.7 & 36.7 & 0.94 & 15 & 20 & S \\
\hline Aserradero & $\mathrm{C} 1$ & $42^{\circ} 28^{\prime} 25.9^{\prime \prime} \mathrm{S}$ & $71^{\circ} 35^{\prime} 11.1^{\prime \prime} \mathrm{O}$ & 543 & 40.4 & 48.4 & 0.90 & 30 & 30 & S \\
\hline Intermedio & $\mathrm{C} 2$ & $42^{\circ} 28^{\prime} 55.9^{\prime \prime} \mathrm{S}$ & $71^{\circ} 33^{\prime} 47.2^{\prime \prime} \mathrm{O}$ & 542 & 45.0 & 46.0 & 1.07 & 32 & 33 & $S$ \\
\hline Bajada/cruce & $\mathrm{C} 3$ & $42^{\circ} 29^{\prime} 17.2^{\prime \prime} \mathrm{S}$ & 71³3’15.6” O & 537 & 52.4 & 63.7 & 0.80 & 23 & 29 & S \\
\hline Puesto & $\mathrm{C} 4$ & $42^{\circ} 29^{\prime} 51.4^{\prime \prime} \mathrm{S}$ & $71^{\circ} 32^{\prime 2} 23.3^{\prime \prime} \mathrm{O}$ & 537 & 52.1 & 57.1 & 2.44 & 37 & 33 & $S$ \\
\hline Camping & C5 & $42^{\circ} 30^{\prime} 11.8^{\prime \prime} \mathrm{S}$ & 71³1'35.7'” O & 534 & 39.5 & 41.5 & 1.23 & 33 & 28 & M \\
\hline La frontera & C6 & $42^{\circ} 31^{\prime} 27.6^{\prime \prime} \mathrm{S}$ & $71^{\circ} 31^{\prime} 58.8^{\prime \prime} \mathrm{O}$ & 533 & 42.1 & 56.1 & 1.46 & 22 & 38 & S \\
\hline Balsa Murmullos & C7 & $42^{\circ} 32^{\prime} 12.3^{\prime \prime} \mathrm{S}$ & $71^{\circ} 33^{\prime} 35.2^{\prime \prime} \mathrm{O}$ & 529 & 49.3 & 76.5 & 0.97 & 10 & 8 & S \\
\hline Villa Rivadavia & $\mathrm{C} 8$ & $42^{\circ} 33^{\prime} 20.3^{\prime \prime} \mathrm{S}$ & $71^{\circ} 36^{\prime} 02.5^{\prime \prime} \mathrm{O}$ & 527 & 50.0 & 60.3 & $\mathrm{~S} / \mathrm{D}$ & 30 & 17 & M \\
\hline La Bolsa & C9 & $42^{\circ} 33^{\prime} 30.4^{\prime \prime} \mathrm{S}$ & $71^{\circ} 37^{\prime} 18.5^{\prime \prime} \mathrm{O}$ & 529 & 53.0 & 56.0 & $S / D$ & 32 & 30 & S \\
\hline Desembocadura & C10 & $42^{\circ} 33^{\prime} 43.8^{\prime \prime} \mathrm{S}$ & $71^{\circ} 38^{\prime} 03.0^{\prime \prime} \mathrm{O}$ & 528 & 71.0 & 81.4 & $\mathrm{~S} / \mathrm{D}$ & 14 & 15 & S \\
\hline
\end{tabular}



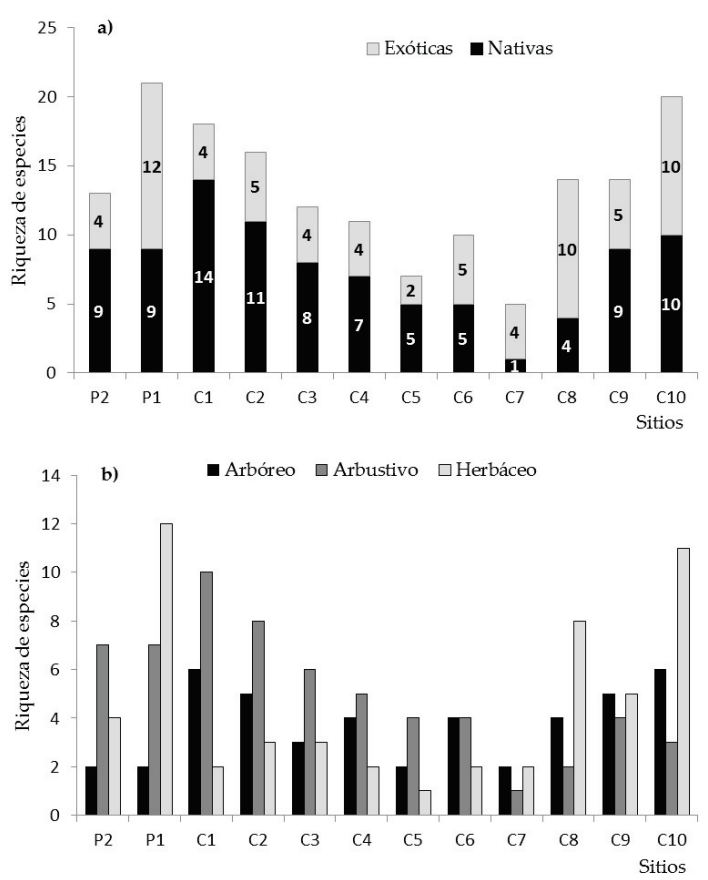

Figura 2. Riqueza de plantas ribereñas en el total de tramos analizados, según: a) hábito (árbol, arbusto, hierba) y b) origen (nativa, exótica). Referencias: $C=$ Carrileufu, $\mathrm{P}=$ Pedregoso.

Figure 2. Riparian plants richness in all the studied stretches according to the: a) habit (tree, bush, herb) and $b$ ) origin (native, exotic). References: $C=$ Carrileufu, $\mathrm{P}=$ Pedregoso. proporción de especies arbóreas, arbustivas y herbáceas en los distintos tramos relevados (Figura 2).

Entre los atributos de la comunidad vegetal de cada tramo, la riqueza de especies resultó mayor en el sitio P1 (21 especies), ubicado sobre el río Pedregoso, y los sitios C1 (18 especies) y C10 (20 especies) del río Carrileufu. En los dos primeros tramos del río Carrileufu se registró la mayor cantidad de plantas nativas, con el 78\% y $69 \%$ de especies, respectivamente. La mayor variación en los valores de diversidad se presentó en tramos del río Carrileufu (Tabla 3). Los sitios P1 y C10 presentaron una riqueza de especies similar, con una mayor equitatividad en C10, lo que contribuyó al aumento de su diversidad. En P1 se registró el ñire como especie dominante, seguido por especies del género Poa. En el caso de C3, la baja diversidad puede explicarse por una riqueza específica intermedia y una alta dominancia de ñire (65\% de cobertura); en cambio, en C7 se observó una baja riqueza de especies, con el lupino como especie dominante y en menor proporción el ñire y el sauce (Anexo 1).

Los valores del índice QBRp estuvieron comprendidos entre 62 (C7) y 92.5 (C2), correspondiendo a calidad de ribera

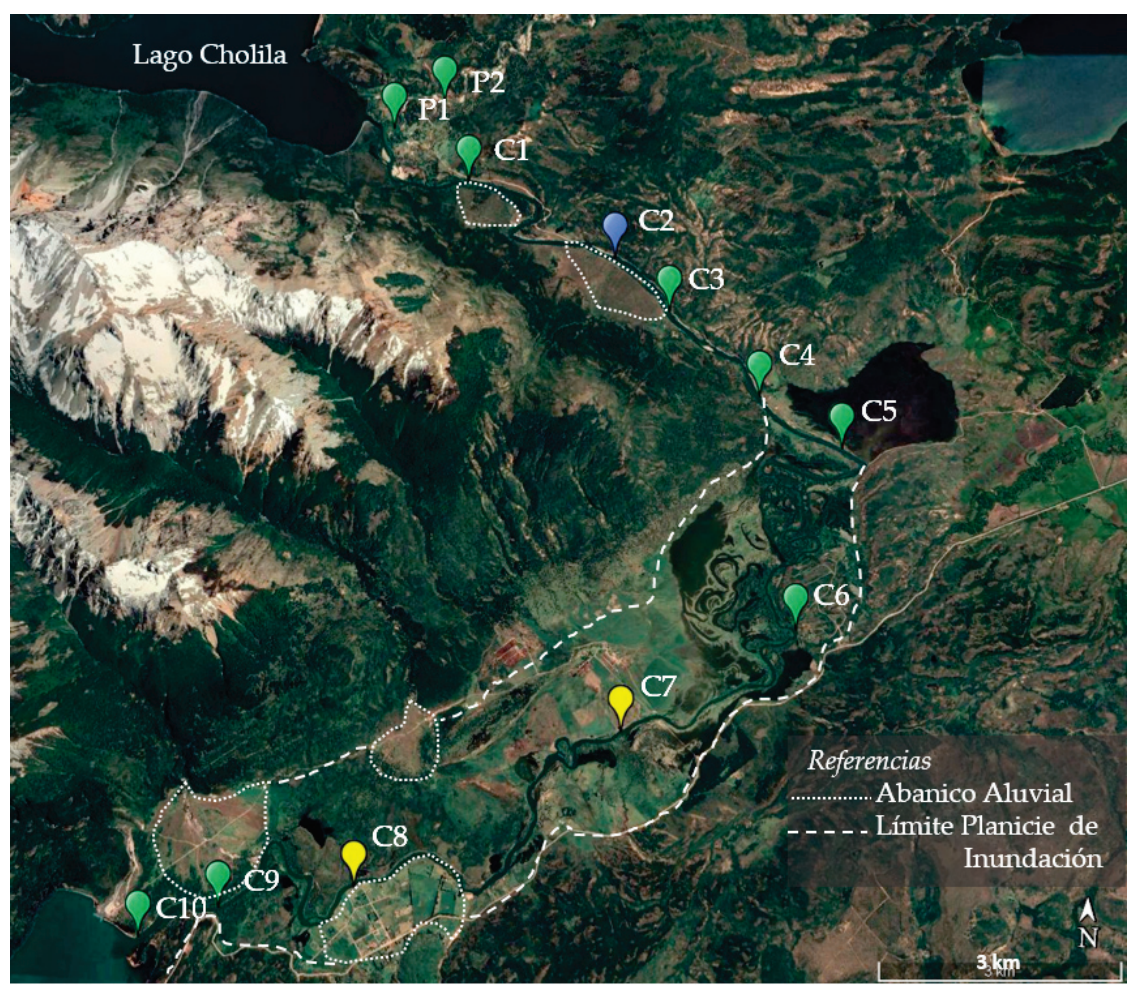

Figura 3. Imagen satelital de Google Earth Pro versión 7.3.2 con la ubicación de los sitios estudiados y valoración de la calidad de ribera, indicada con el color que establece el índice QBRp (ver Tabla 4). Se indican las principales geoformas.

Figure 3. Satellite image from Google Earth Pro version 7.3.2 with the location of the studied sites and the assessment of the riparian quality, indicated with the color established by the QBRp index (see Table 4). The main geoforms are indicated. 
Tabla 3. Atributos de la comunidad ribereña (riqueza específica, diversidad $\mathrm{H}^{\prime}$, equitatividad J') para cada tramo de los ríos Pedregoso y Carrileufu (provincia de Chubut, Argentina).

Table 3. Riparian community attributes (species richness, diversity $\mathrm{H}^{\prime}$, equability $\mathrm{J}^{\prime}$ ) of the different stretches from Pedregoso and Carrileufu rivers (province of Chubut, Argentina).

\begin{tabular}{lcccccccccccc}
\hline Atributos ecológicos & P2 & P1 & C1 & C2 & C3 & C4 & C5 & C6 & C7 & C8 & C9 & C10 \\
\hline Riqueza de especies & 13 & 21 & 18 & 16 & 12 & 11 & 7 & 10 & 5 & 14 & 14 & 20 \\
$\begin{array}{l}\text { Índice de diversidad } \\
\text { de Shannon H' }\end{array}$ & 1.93 & 1.86 & 2.14 & 1.71 & 1.42 & 1.64 & 1.47 & 1.42 & 1.30 & 1.85 & 1.83 & 2.17 \\
$\begin{array}{l}\text { Equitatividad de } \\
\text { Shannon J' }\end{array}$ & 0.84 & 0.81 & 0.93 & 0.74 & 0.62 & 0.71 & 0.64 & 0.62 & 0.57 & 0.80 & 0.80 & 0.94 \\
\hline
\end{tabular}

Tabla 4. Valores totales y por apartado del índice QBRp, y usos de la tierra en cada tramo de los ríos Pedregoso y Carrileufu (provincia de Chubut, Argentina).

Table 4. Total and per section values of the QBRp index, and land uses in each stretch of the Pedregoso and Carrileufu rivers (Province of Chubut, Argentina).

\begin{tabular}{|c|c|c|c|c|c|c|c|}
\hline Sitio & 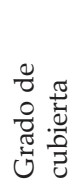 & 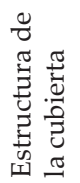 & 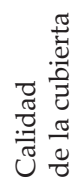 & $\begin{array}{l}\frac{\pi}{\pi} \\
\frac{\pi}{0} \\
\frac{\pi}{\pi} \\
\stackrel{\pi}{Z} \\
\frac{\pi}{Z}\end{array}$ & QBRp & Nivel de calidad & Usos antrópicos \\
\hline P2 & 20 & 15 & 15 & 20 & 70 & \multirow{3}{*}{$\begin{array}{l}\text { Bosque ligeramente } \\
\text { perturbado. Buena } \\
\text { calidad }\end{array}$} & $\begin{array}{l}\text { Ganadero (vacuno) } \\
\text { Extracción de áridos }\end{array}$ \\
\hline P1 & 22 & 21 & 15 & 25 & 83 & & $\begin{array}{l}\text { Ganadero (ovino) } \\
\text { Turístico }\end{array}$ \\
\hline $\mathrm{C} 1$ & 25 & 17 & 22.5 & 25 & 89.5 & & $\begin{array}{l}\text { Ganadero (ovino) } \\
\text { Aserradero-Turístico }\end{array}$ \\
\hline $\mathrm{C} 2$ & 25 & 20 & 22.5 & 25 & 92.5 & $\begin{array}{l}\text { Bosque sin } \\
\text { alteraciones. Calidad } \\
\text { muy buena, estado } \\
\text { natural }\end{array}$ & Ganadero (vacuno) \\
\hline $\mathrm{C} 3$ & 20 & 20 & 20 & 25 & 85 & \multirow{4}{*}{$\begin{array}{l}\text { Bosque ligeramente } \\
\text { perturbado. Buena } \\
\text { calidad }\end{array}$} & $\begin{array}{l}\text { Ganadero (vacuno) } \\
\text { Turístico }\end{array}$ \\
\hline $\mathrm{C} 4$ & 25 & 17 & 17.5 & 25 & 84.5 & & $\begin{array}{l}\text { Ganadero (vacuno) } \\
\text { Turístico }\end{array}$ \\
\hline C5 & 17 & 20 & 15 & 25 & 77 & & Turístico \\
\hline C6 & 25 & 20 & 15 & 25 & 85 & & $\begin{array}{l}\text { Urbano-Ganadero } \\
\text { (ovino, vacuno) }\end{array}$ \\
\hline $\mathrm{C} 7$ & 17 & 15 & 10 & 20 & 62 & \multirow{2}{*}{$\begin{array}{l}\text { Inicio de alteración } \\
\text { importante. Calidad } \\
\text { intermedia }\end{array}$} & $\begin{array}{l}\text { Ganadero (vacuno) } \\
\text { Agrícola }\end{array}$ \\
\hline $\mathrm{C} 8$ & 17 & 8 & 12.5 & 25 & 62.5 & & $\begin{array}{l}\text { Urbano rural } \\
\text { Ganadero (vacuno) } \\
\text { Turístico }\end{array}$ \\
\hline C9 & 25 & 18 & 17.5 & 25 & 85.5 & \multirow{2}{*}{$\begin{array}{l}\text { Bosque ligeramente } \\
\text { perturbado. Buena } \\
\text { calidad }\end{array}$} & $\begin{array}{l}\text { Turístico-Ganadero } \\
\text { (vacuno) }\end{array}$ \\
\hline $\mathrm{C} 10$ & 25 & 20 & 17.5 & 25 & 87.5 & & $\begin{array}{l}\text { Turístico-Ganadero } \\
\text { (vacuno) }\end{array}$ \\
\hline
\end{tabular}

intermedia a muy buena (Tabla 4, Figura 3), no registrándose tramos del río con calidad mala o pésima. Los dos tramos del arroyo Pedregoso presentaron un bosque ribereño ligeramente perturbado, con una calidad buena. En el río Carrileufu se registró un solo tramo cuya ribera presentó un estado natural y muy buena calidad; dos tramos con inicio de alteración importante y riberas de calidad intermedia, y los siete tramos restantes presentaron bosques de ribera ligeramente perturbados, con buena calidad.
En relación con los usos de la tierra, en todos los tramos se registró al menos un tipo de uso. El $66.7 \%$ de los tramos presentó dos usos diferentes, un $16.7 \%$ sólo uno, y el otro $16.7 \%$ presentó tres usos diferentes. En general, el uso más extendido fue el ganadero (ovino y/o bovino), en la mayoría de los casos desarrollado de manera extensiva, con excepción del sitio C7 (estancia Los Murmullos), que presenta una actividad agropastoril importante con pasturas, ganadería semi-intensiva y ganadería intensiva en la modalidad feedlot. 
Por las bellezas escénicas, muchos de los sitios presentan actividades recreativo-turísticas y sólo en algunos tramos se registran actividades como extracción forestal y de leña, extracción de áridos y asentamientos poblacionales (Tabla 4).

A partir del análisis Hill-Smith se ordenaron los distintos sitios en función de las variables físicas, calidad de los bosques ribereños y los usos de la tierra. Los tres primeros ejes explicaron el $66.25 \%$ de la varianza total, con las variables QBRp, estructura de la cubierta vegetal y pendiente de ribera izquierda que aportaron en mayor medida en el primer componente; cobertura total y suelo desnudo aportaron más en el segundo componente, mientras que pendiente ribera derecha, altitud y ancho mojado lo hicieron en el tercer componente. La variable altitud aportó en la misma magnitud (0.5) en los tres componentes. Se observó una relación inversa entre la calidad de los bosques de ribera y la sinergia o superposición de usos (Figura 4). A

Tabla 5. Factibilidad de restauración y tipo de manejo para cada uno de los tramos de los ríos Pedregoso y Carrileufu (Chubut, Argentina). Referencias: (1) Extracción forestal y de áridos; (2) Tomas de agua, canales de riego.

Table 5. Restoration feasibility and management perspectives for each stretch of the Pedregoso and Carrileufu rivers (Chubut, Argentina). Ref.: (1) Forest and sediment/aggregate extraction; (2) Water intakes, irrigation channels.

\begin{tabular}{|c|c|c|c|c|c|c|c|c|c|c|c|c|c|}
\hline : & 商 & $\begin{array}{l}\frac{0}{0} \\
\frac{\pi}{0} \\
\frac{5}{20} \\
\frac{\pi}{0} \\
\frac{0}{\pi} \\
\frac{\pi}{7}\end{array}$ & 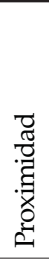 & 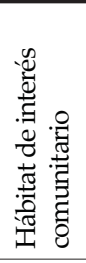 & 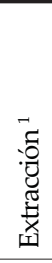 & 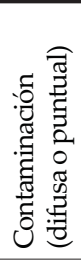 & 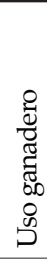 & 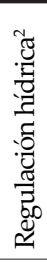 & 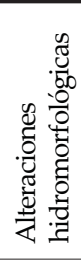 & 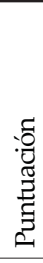 & 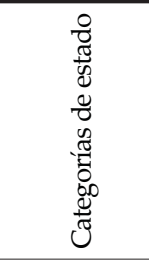 & 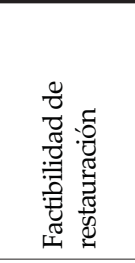 & 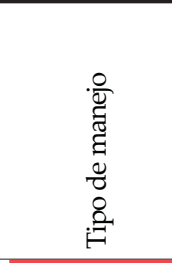 \\
\hline $\mathrm{P} 2$ & 70 & 3 & & & -1 & & -1 & & -1 & 0 & $\begin{array}{c}\text { Estado } \\
\text { deficiente }\end{array}$ & Difícil & Rehabilitación \\
\hline P1 & 83 & 4 & +1 & +1 & & & -1 & -1 & & 4 & $\begin{array}{c}\text { Estado } \\
\text { Intermedio }\end{array}$ & Moderada & Restauración \\
\hline $\mathrm{C} 1$ & 89.5 & 4 & +1 & & -1 & & -1 & -1 & & 2 & $\begin{array}{c}\text { Estado } \\
\text { Intermedio }\end{array}$ & Difícil & $\begin{array}{l}\text { Restauración } \\
\text { compleja/ } \\
\text { Revegetación }\end{array}$ \\
\hline $\mathrm{C} 2$ & 92.5 & 5 & +1 & & & & -1 & & & 5 & $\begin{array}{l}\text { Estado } \\
\text { Bueno }\end{array}$ & Fácil & $\begin{array}{l}\text { Conservación/ } \\
\text { Regeneración }\end{array}$ \\
\hline $\mathrm{C} 3$ & 85 & 4 & & +1 & & & -1 & & & 4 & $\begin{array}{c}\text { Estado } \\
\text { Intermedio }\end{array}$ & Moderada & Restauración \\
\hline $\mathrm{C} 4$ & 84.5 & 4 & +1 & & & & -1 & & & 4 & $\begin{array}{c}\text { Estado } \\
\text { Intermedio }\end{array}$ & Moderada & Restauración \\
\hline C5 & 77 & 4 & +1 & & & & & & & 5 & $\begin{array}{l}\text { Estado } \\
\text { Bueno }\end{array}$ & Fácil & $\begin{array}{l}\text { Conservación/ } \\
\text { Regeneración }\end{array}$ \\
\hline C6 & 85 & 4 & & & & -1 & -1 & -1 & & 1 & $\begin{array}{l}\text { Estado } \\
\text { deficiente }\end{array}$ & Difícil & Rehabilitación \\
\hline $\mathrm{C} 7$ & 62 & 3 & & & & -1 & -1 & -1 & & 0 & $\begin{array}{l}\text { Estado } \\
\text { deficiente }\end{array}$ & Difícil & Rehabilitación \\
\hline $\mathrm{C} 8$ & 62.5 & 3 & & & & -1 & -1 & -1 & & 0 & $\begin{array}{c}\text { Estado } \\
\text { deficiente }\end{array}$ & Difícil & Rehabilitación \\
\hline C9 & 85.5 & 4 & & & & & -1 & & & 3 & $\begin{array}{c}\text { Estado } \\
\text { Intermedio }\end{array}$ & Moderada & Restauración \\
\hline C10 & 87.5 & 4 & +1 & +1 & & & -1 & & & 5 & $\begin{array}{l}\text { Estado } \\
\text { Bueno }\end{array}$ & Fácil & $\begin{array}{l}\text { Conservación/ } \\
\text { Regeneración }\end{array}$ \\
\hline
\end{tabular}




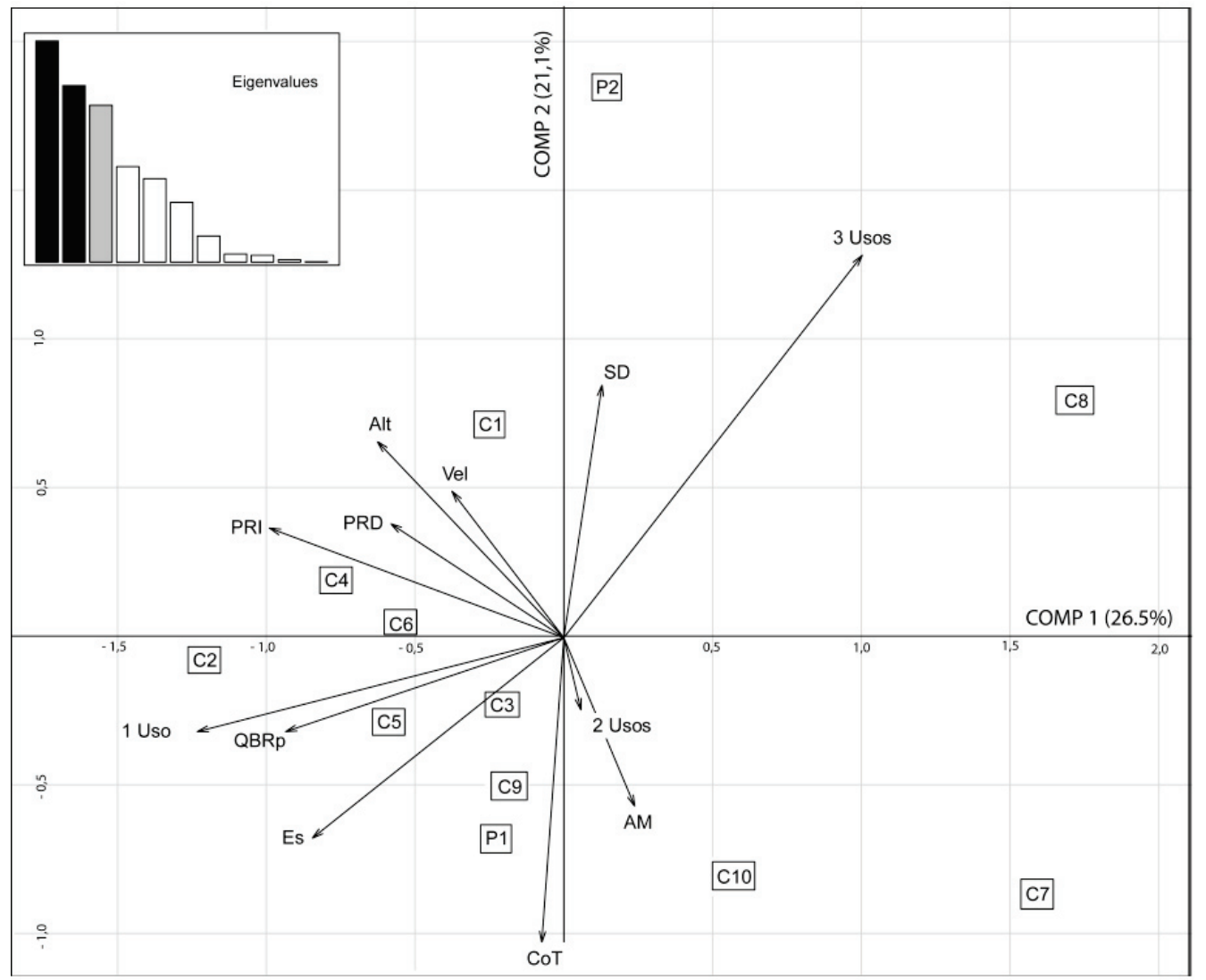

Figura 4. Ordenamiento de los tramos de los ríos Pedregoso y Carrileufu en relación con variables físicas, valores del índice QBRp y usos de la tierra, obtenido a partir de un análisis Hill-Smith.

Figure 4. Ordination of the stretches of the Carrileufu and Pedregoso rivers in relation to physical variables, QBRp index values and land uses, obtained from a Hill-Smith analysis.

mayor superposición de usos de la tierra en un tramo determinado del río correspondió un menor valor del QBRp, de la estructura de la vegetación y de la pendiente de ribera izquierda, como así también una menor cobertura total de plantas, lo cual se asoció con una mayor proporción de suelo desnudo.

El análisis conjunto del índice QBRp y la afectación de cada tramo del río por los distintos usos de la tierra permitió establecer tres tramos con categoría de estado Bueno, cinco con Intermedio y cuatro con estado Deficiente (Tabla 5). A partir de estos últimos datos se determinó la factibilidad de restauración para cada sitio, con los tramos P2 y C6 a C8 clasificados como difícil. El resto de los tramos alternaron entre fácil y moderada. El tipo de manejo recomendado incluyó desde la conservación/regeneración (C2, C5 y C10), a la rehabilitación (P2, C6-C8) y la restauración (P1, C3, C4 y C9), con el tramo C1 donde se recomienda la restauración compleja/ revegetación.

\section{DisCUSIÓN}

Los valores del índice QBRp para los distintos tramos del río Carrileufu y su afluente río Pedregoso muestran un patrón general caracterizado por una buena calidad de los bosques de ribera en los primeros y los últimos tramos del sistema fluvial. Indican, además, una relación inversa entre la calidad de los bosques ribereños y la superposición e intensidad de usos de la tierra, lo cual quedó reflejado en el análisis Hill-Smith, en el que se observa el ordenamiento de los distintos tramos del río en función de las variables ecológicas y ambientales consideradas. Más allá de los procesos que ocurren naturalmente en el río, los cambios en el uso de la tierra quedaron en evidencia, por ejemplo, en el incremento de sedimentos finos en el lecho del río, principalmente en los tramos medios, por erosión de los suelos expuestos por efecto de actividades extractivas y agropecuarias, construcción de caminos y urbanizaciones. Esto coincide con trabajos previos en la 
región andino-patagónica, en los que se estableció que las consecuencias más severas sobre el corredor ribereño fueron en sitios con pasturas y urbanizados (Kutschker et al. 2009; Papazian 2009; Miserendino et al. 2011). Estas intervenciones en el territorio modificaron y fragmentaron el paisaje tanto a escala de cuenca como a escala local, lo que afectó de manera directa o indirecta la calidad de los bosques de ribera y el estado ecológico del ecosistema fluvial asociado debido a las múltiples interacciones existentes entre los ecosistemas acuáticos y los terrestres (Ward 1989).

Los dos tramos estudiados del río Pedregoso presentaron sus bosques de ribera ligeramente perturbados, con una buena calidad, aunque registraron diferencias enla estructura y calidad de la vegetación y en la naturalidad del canal fluvial, lo que evidencia, fundamentalmente, los efectos negativos de las actividades extractivas. Ambos sitios se localizan en el segmento inferior de la faja de meandros del río donde ha desarrollado su planicie aluvial y algunos bancos de sedimentos. En particular en el primer tramo (P2), este material constituye una fuente utilizada para la extracción de áridos, actividad que modifica la dinámica del río y consecuentemente de sus riberas. Estas modificaciones estacionales en el cauce y las márgenes del río condicionan el establecimiento de la vegetación y, aunque sus riberas presentaron una menor riqueza de plantas en relación al siguiente tramo (P1), se registró una mayor proporción de especies nativas y una mayor diversidad, con una comunidad vegetal más equitativa.

La mayoría de los tramos del Carrileufu también presentaron riberas mínimamente alteradas, de buena calidad; sólo un tramo presentó un bosque sin perturbaciones y una calidad de ribera muy buena, en estado natural, mientras que dos tramos ubicados en la parte media-baja del sistema fluvial se caracterizaron por un inicio de alteración importante y una calidad de ribera intermedia. No se registraron riberas con mala o pésima calidad, lo cual podría deberse a que los usos históricos del territorio se realizan mayormente de manera extensiva o a pequeña escala y, por otro lado, a que las riberas, si bien varían en cobertura, estructura y calidad de sus bosques, mantienen en general una buena conectividad longitudinal, lo que contribuye a la conservación de las funciones y procesos del ecosistema ribereño como la estabilización del sustrato y la dispersión de semillas, entre otros.

En sectores sometidos a varios usos de la tierra (como el primer tramo del río Carrileufu [C1-Aserradero]), si bien presentó uno de los valores más altos de diversidad de especies y de calidad de bosques de ribera, es un sector con ganadería extensiva, actividades turísticas y el único tramo donde se registró extracción forestal. Diversos estudios en Nueva Zelanda sobre el efecto que pueden tener las actividades forestales sobre los ríos postulan que las distintas prácticas forestales como plantación, mantenimiento y cosecha pueden resultar en modificaciones serias sobre estos ecosistemas (Harding 2003). La tala y el desmonte previos a la plantación o a la extracción de la madera degradan los suelos de las laderas adyacentes al curso de agua, lo cual provoca un aumento significativo en la entrada de sedimentos al cauce (Quinn et al. 2004). Esto ocurre en el área de estudio, aun cuando la Ley de Ordenamiento de Bosques Nativos de la provincia de Chubut (Ley XVII $\mathrm{N}^{\circ}$ 92/2010) contempla la conservación de los bosques de protección de las riberas de los ríos (corredores ribereños) con el fin de evitar cambios en los procesos y en los regímenes naturales de los mismos.

Lostramosdondesedesarrollóinfraestructura y urbanizaciones correspondieron a los de menor calidad de sus bosques de ribera (C7Los Murmullos y C8-Villa Lago Rivadavia), pero en estos casos, la superposición de usos contribuyó de manera negativa en la calificación. El primero de esos tramos presentó la menor riqueza y diversidad de plantas, como así también el menor valor del índice QBRp, lo que indica riberas de calidad intermedia. Este sector incluye una amplia extensión de tierras adyacentes al río dedicadas a la actividad agrícola y a la ganadera intensiva, que si bien mantiene una delgada faja de vegetación ribereña más o menos continua, está conformada, en general, por especies exóticas. Esta transformación de bosques nativos en pasturas afecta las cuencas de drenaje, y esto repercute en las condiciones típicas de cada río (Rood et al. 2014); por otro lado, la presencia de ganado afecta la condición de las riberas a partir de la compactación del suelo por pisoteo, forrajeo y reducción de la regeneración de la vegetación y deposición de excretas, lo cual influye de manera directa en la calidad ecológica del ecosistema acuático (Gantes et al. 2014). El otro tramo 
urbanizado corresponde a un asentamiento poblacional ubicado en la margen izquierda del río y asentado sobre un abanico aluvial, en el cual se registró, además, uso ganadero y actividades turísticas. Una condición diferente se observó en el sitio La Frontera (C6), que también constituye un sector urbanizado con un loteo tipo country, donde se registró una buena calidad de sus riberas. Sin embargo, se vio afectada la cobertura y riqueza de especies, así como la estructura y calidad de la cubierta vegetal al suprimir distintos estratos de especies nativas, como resultado de las tareas de mantenimiento del terreno (parquización) incluidas las riberas. Frente a este proceso de urbanización son varias las consecuencias que sufre el río, entre ellas la fragmentación del paisaje, con disminución de cobertura arbórea y arbustiva y la consecuente pérdida de sustrato por el incremento de los procesos erosivos. Esto último, a su vez, conduce a modificaciones en la morfología del cauce, al alterarse los pulsos de erosiónsedimentación (Vizcaíno et al. 2003). En este contexto se ven afectadas sustancialmente las múltiples funciones, bienes y servicios que brindan los ecosistemas ribereños (Ceccon 2003; Elosegi and Diez 2009; González del Tánago et al. 2012; Valero et al. 2014).

Muchas de las funciones de la vegetación ribereña se mantienen en algunos de los tramos del río Carrileufu, específicamente en los sitios con bosques no alterados o aquellos ligeramente perturbados, donde si bien los valores de riqueza y diversidad de especies difieren en uno y otro, el nivel de cobertura y la estructura de la cubierta vegetal permiten el desarrollo de una ribera de calidad muy buena o buena. En estos sectores se determinó una factibilidad de restauración de fácil a moderada, con acciones de manejo que incluyen la regeneración/ conservación y restauración. El tramo P2 del arroyo Pedregoso, así como los tramos C6, C7 y C8 del río Carrileufu fueron clasificados con un estado deficiente de sus riberas, y una factibilidad de restauración difícil, que requiere de acciones de rehabilitación para su recuperación. Un ecosistema de ribera continuo y con una dimensión transversal mínima desde cabecera a desembocadura es imprescindible para garantizar la viabilidad y funcionalidad del ecosistema de ribera (García Quiroga and Abad Soria 2014).

Este estudio rescata la importancia de mantener la integridad de los bosques de ribera como condición para preservar el estado ecológico de los ecosistemas fluviales y para minimizar los efectos de la intensificación de los disturbios de origen antrópico que los afecta de forma directa o indirecta. Se ha comprobado que los cambios en el uso del suelo conducen a la pérdida de vegetación natural y la homogeneización o simplificación estructural de los ecosistemas de ribera, contribuyendo a su erosión y degradación (Allan 2004; Miserendino et al. 2011; Cisneros et al. 2012). En ese sentido, el índice de calidad de bosques de ribera que se aplicó previamente en ríos andino-patagónicos (QBRp) demostró ser una herramienta rápida y eficaz para evaluar y monitorear los aspectos relacionados con la estructura y la calidad de la vegetación ribereña. Por otro lado, las categorías de estado reflejan las consecuencias de las intervenciones en el territorio a una escala más amplia que la escala de tramo que evalúa el QBRp, aportando un abordaje integral de estos ambientes y más elementos de análisis para definir su factibilidad de restauración. Como fuera señalado en otros estudios (Kauffman et al. 1997; Stromberg 2001; Meli et al. 2013; Valero et al. 2014), se sugieren diferentes tipos de manejo para la recuperación ecológica de estos ecosistemas de ribera.

El mantenimiento del bosque ribereño se ha convertido en un componente integral de las estrategias de manejo para la conservación de la biodiversidad (Naiman and Décamps 1997). Es por ello que resulta de fundamental relevancia sostener una buena calidad de los bosques de ribera en el río Carrileufu y sus tributarios, pues constituye la vía de ingreso fluvial a un Parque Nacional. El tratamiento del espacio del río y su entorno a partir del análisis multicriterio aquí aplicado complementa la evaluación ecológica de los bosques de ribera. Se demuestra la utilidad de otra herramienta de gestión para intervenir tanto a escala local (tramo del río) como a escala de cuenca o paisaje, con el fin de recuperar el rol multifuncional de los ecosistemas de ribera, así como garantizar la compatibilidad entre los usos actuales y los objetivos de preservación, uso sustentable y mitigación de los ambientes degradados, inherentes a la conservación de la naturaleza.

Agradecimientos. A los encargados del camping Lago Cholila, del barrio La Frontera y el establecimiento Los Murmullos, por permitirnos acceder a sus predios para llevar adelante los muestreos a campo. A M. L. Miserendino por la lectura crítica del manuscrito y a los revisores por sus valiosos aportes que contribuyeron a la mejora del trabajo. 


\section{REFERENCIAS}

Allan, J. D. 2004. Landscapes and riverscapes: The Influence of Land Use on Stream Ecosystems. Annu Rev Ecol Evol Syst 35:257-84. https://doi.org/10.1146/annurev.ecolsys.35.120202.110122.

Boudell, J. A., M. D. Dixon, S. B. Rood, and J. C. Stromberg. 2015. Restoring functional riparian ecosystems: concepts and applications. Ecohydrology 8(5):747-752. https://doi.org/10.1002/eco.1664.

Brice, J. C. 1964. Channel patterns and terraces of the Loup Rivers. US Geological Survey Professional Paper, Nebraska, 422-D. https://doi.org/10.3133/pp422D.

Calderón, M. 2007. Hidrología. Plan Estratégico de Manejo Conjunto de las áreas este y norte linderas con el Parque Nacional Los Alerces, Chubut - Argentina. Informe Final-CFI.

Ceccon, E. 2003. Los bosques ribereños y la restauración y conservación de las cuencas hidrográficas. Ciencias 72: 46-53.

Cisneros, J., C. Cholaky, A. Cantero Gutiérrez, J. González, M. Reynero, A. Diez, and L. Bergesio. 2012. Erosión hídrica. Principios y técnicas de manejo. Ed. UniRío, Córdoba. Pp. 290.

Confederación Hidrográfica del Segura (CHS). 2008. Restauración de riberas. Manual para la restauración de Riberas en la cuenca del Río Segura. Ed. Ministerio de Medio Ambiente y medio Rural y Marino. Pp. 230.

Correa, M. O. (dir.). 1978-1999. Flora Patagónica. Tomo VIII: parte I, II, III, IVa, IVb, V, y VIII. Colección científica del INTA, Buenos Aires.

DeFries, R. S., J. A. Foley, and G. P. Asner. 2004. Landロuse choices: balancing human needs and ecosystem function. Front Ecol Environ 2:249-257. https:/ /doi.org/10.1890/1540-9295(2004)002[0249:LCBHNA]2.0.CO;2.

Dudgeon, D., A. H. Arthington, M. O. Gessner, Z. Kawabata, D. J. Knowler, C. Lévêque, R. J. Naiman, A. PrieurRichard, D. Soto. M. L. J. Stiassny, and C. A. Sullivan. 2006. Freshwater biodiversity: importance, threats, status and conservation challenges. Biol Rev 81:163-182. https://doi.org/10.1017/S1464793105006950.

Elosegi, A., and J. Díez. 2009. La vegetación terrestre asociada al río: el bosque de ribera. Pp. 311-321 en A. Elosegi and S. Sabater (eds.). Conceptos y técnicas en ecología fluvial. Fundación BBVA, España.

Ezcurra, C., and C. A. M. Brion. 2005. Plantas del Nahuel Huapi. Catálogo de la Flora Vascular del Parque Nacional Nahuel Huapi, Argentina. Ed. Bavaria, San Carlos de Bariloche. Pp. 1-70.

Fernández, R. D., S. J. Ceballos, A. L. González Achem, M. V. Hidalgo, and H. R. Fernández. 2016. Quality and Conservation of Riparian Forest in a Mountain Subtropical Basin of Argentina. International Journal of Ecology. Artículo ID 4842165. Pp. 10. https:/ / doi.org/10.1155/2016/4842165.

Gantes, P., L. Falco, and A. Sánchez Caro. 2014. Efecto del ganado sobre la estructura de la vegetación en las riberas de un arroyo pampeano. Biología Acuática 30:123-131.

García Quiroga, F., and J. Abad Soria. 2014. Los corredores ecológicos y su importancia ambiental: Propuestas de actuación para fomentar la permeabilidad y conectividad aplicadas al entorno del río Cardeña (Ávila y Segovia). Observatorio Medioambiental 17:253-298. https://doi.org/10.5209/rev_OBMD.2014.v17.47194.

González del Tánago, M., D. García de Jalón, and M. Román. 2012. River restoration in Spain: Theoretical and practical approach in the context of the European Water Framework Directive. Environ Manage 50(1):123-139. https://doi.org/ 10.1007/s00267-012-9862-1.

Gordon, N. D., T. A. McMahon, B. L. Finlayson, C. J. Gippel, and R. J. Nathan. 2004. Stream hydrology. An introduction for ecologists. John Wiley and Sons Ltd. Sussex, England, 2nd edition. Pp. 429.

Gregory, S. V., F. J. Swanson, W. A. McKee, and K. W. Cummins. 1991. An ecosystem perspective of riparian zones. BioScience 41:540-551. https:/ / doi.org/10.2307/1311607.

Harding, J. S. 2003. Historic deforestation and the fate of endemic invertebrate species in streams. New Zeal J Mar Fresh 37:333-345. https:/ / doi.org/10.1080/00288330.2003.9517171.

HIDRI (Agència Catalana de l'Aigua). 2006. Protocolo para la valoración de la calidad hidromorfológica de los ríos. España.

Hill, M. O., and A. J. E. Smith. 1976. Principal component analysis of taxonomic data with multi-state discrete characters. Taxon 25:249-255. https://doi.org/10.2307/1219449.

Kauffman, J. B., R. L. Beschta, N. Otting, and D. Lytjen. 1997. An ecological perspective of riparian and stream restoration in the western United States. Fisheries 22(5):12-24. https:/ / doi.org/10.1577/1548-8446(1997)022\%3C0012: AEPORA\%3E2.0.CO;2.

Kutschker, A.; C. Brand, M. L. Miserendino. 2009. Evaluación de la calidad de los bosques de ribera en ríos del NO del Chubut sometidos a distintos usos de la tierra. Ecología Austral 19:19-34.

MacFarlane, W. W., J. T. Gilbert, J. D. Gilbert, W. C. Saunders, N. Hough-Snee, C. Hafen, J. M. Wheaton, and S. N. Bennett. 2018. What are the conditions of riparian ecosystems? Identifying impaired floodplain ecosystems across the western U.S. using the Riparian Condition Assessment (RCA) tool. Environ Manage 62:548-570. https: / doi.org/ 10.1007/s00267-018-1061-2.

Mander, O., Y. Hayakawa, and V. Kuusemets. 2005. Purification processes, ecological functions, planning and design of riparian buffer in agricultural watersheds. Ecol Eng 24:421-432. https://doi.org/10.1016/j.ecoleng.2005.01.015.

Martínez, O. A. 2007. Geología y Geomorfología. Plan Estratégico de Manejo Conjunto de las áreas este y norte linderas con el Parque Nacional Los Alerces, Chubut - Argentina. Informe Final CFI.

Maurín, J. D. 2014. Propuesta para la determinación de la Línea de Ribera en San Juan. Fiscalía de Estado de la Provincia de San Juan. Pp. 243-260. 
Meli, P., M. Martínez-Ramos, and J. M. Rey-Benayas. 2013. Selecting species for passive and active riparian restoration in Southern Mexico. Restor Ecol 21(2):163-165. https:/ / doi.org/10.1111/j.1526-100X.2012.00934.x.

Miserendino, M. L., C. Brand, and C. Y. Di Prinzio. 2008. Assessing Urban Impacts on Water Quality, Benthic Communities and Fish in Streams of the Andes Mountains, Patagonia (Argentina). Water Air Soil Poll 194(1):91-110.

Miserendino, M. L., R. Casaux, M. Archangelsky, C. Y. Di Prinzio, C. Brand, and A. M. Kutschker. 2011. Assessing land-use effects on water quality, in-stream habitat, riparian ecosystems and biodiversity in Patagonian Northwest streams. Sci Total Environ 409:572-584. https://doi.org/10.1007/s11270-008-9701-4.

Munné, A., C. Solá, and N. Prat. 1998. QBR: Un índice rápido para la evaluación de la calidad de los ecosistemas de ribera. Tecnología del Agua 175:20-37.

Munné, A., N. Prat, C. Solá, N. Bonada, and M. R. Rieradevall. 2003. A simple field method for assessing the ecological quality of riparian habitat in rivers and streams: QBR index. Aquat Conserv 13:147-163. https://doi.org/10.1002/ aqc.529.

Naiman, R. J., and H. Décamps. 1997. The ecology of interfaces: Riparian Zones. Annu Rev Ecol S 28:621-658. https: //doi.org/10.1146/annurev.ecolsys.28.1.621.

Naiman, R. J., K. L. Fetherston, S. McKay, and J. Chen. 1998. Riparian forests. Pp. 289-323 in R. J. Naiman and R. E. Bilby. River Ecology and Management: Lessons from the Pacific Coastal Ecoregion. New York. https://doi.org/10.1007/9781-4612-1652-0_12.

Papazian, G. 2009. Evaluación de la calidad de los bosques de ribera en el río Percy, Chubut (Argentina). Tesis de Grado, FCN-UNPSJB. Esquel, Chubut. Pp. 61.

Quinn, J. M., L. K. Boothroyd, and B. J. Smith. 2004. Riparian buffers mitigate effects of pine plantation logging on New Zealand streams: 2. Invertebrate communities. Forest Ecol Manag 191(1-3):129-146. https://doi.org/10.1016/ j.foreco.2003.11.013.

Rood, S. B., S. G. Bigelow, M. L. Polzin, K. M. Gill, and C. A. Coburn. 2014. Biological bank protection: trees are more effective than grasses at resisting erosion from major river floods. 8(5):772-779. https://doi.org/10.1002/eco.1544.

Sabater, S., J. C. Donato, A. Giorgi, and A. Elosegi. 2009. El río como ecosistema. Pp. 23-37 en A. Elosegi and S. Sabater (eds.). Conceptos y técnicas en ecología fluvial. Fundación BBVA, España.

Scott, L. N., A. M. Villamagna, and P. L. Angermeier. 2018 A new modeling approach to prioritize riparian restoration to reduce sediment loading in two Virginia river basins. Environ Manage 62(4):721-739. https://doi.org/10.1007/ s00267-018-1078-6.

Society for Ecological Restoration (SER) International, Grupo de trabajo sobre ciencia y políticas. 2004. Principios de SER International sobre la restauración ecológica. URL: www.ser.org.

Sirombra, M. G., and L. M. Mesa. 2012. A method for assessing the ecological quality ofriparian forests in subtropical Andean streams: QBRy index. Ecol Indic 20:324-331. https://doi.org/10.1016/j.ecolind.2012.02.021.

Stella, J. C., P. M. Rodríguez-González, S. Dufour, and J. Bendix. 2013. Riparian vegetation research in Mediterranean climate regions: common patterns, ecological processes, and considerations for management. Hydrobiologia 719(1): 291-315. https:/ / doi.org/10.1007/s10750-012-1304-9.

Stromberg, J. C. 2001. Restoration of riparian vegetation in the South-Western United States: importance of flow regimes and fluvial dynamism. J Arid Environ 49(1):17-34. https://doi.org/10.1006/jare.2001.0833.

Subsecretaría de Recursos Hídricos (SSRH). 2002. Altas Digital de los Recursos Hídricos Superficiales de la República Argentina. URL: www.hidricosargentina.gov.ar/cartografia chubut.pdf.

Swanson, S., D. Kozlowski, R. Hall, D. Heggem, and J. Lin. 2017. Riparian proper functioning condition assessment to improve watershed management for water quality. J Soil Water Conserv 72(2):168-182. https://doi.org/10.2489/ jswc.72.2.168.

Valero, E., J. Picos, and X. Álvarez. Characterization of riparian forest quality of the Umia River for a proposed restoration. Ecol Eng 67:216-222. https://doi.org/10.1016/j.ecoleng.2014.03.084.

Vizcaíno, P., F. Magdaleno, A. Seves, S. Merino, M. González del Tánago, and D. García de Jalón. 2003. Los cambios geomorfológicos del río Jarama como base para su restauración. Limnetica 22(3-4):1-8.

Ward, J. V. 1989. The four dimensional mature of lotic ecosystems. J North Am Benth Soc 8(1):2-8. https://doi.org/ $10.2307 / 1467397$.

Zuloaga, F., O. Morrone, and M. Belgrano (eds.). 2008. Catálogo de las plantas vasculares del Cono Sur. Ann Miss Bot Gard 107:1-3486. URL: www.darwin.edu.ar/Proyectos/FloraArgentina. 